15. Fukuda H., Mutoh N., Nakamura G., Schattschneider D. A method to generate Polyominoes and Polyamonds for tilings with rotational symmetry. Berlin: Graphs ang Combinatirics, 23, 2007. 259-267.

16. Gardner M. Ch. 11. Polygexes and Polyaboloes. NY.: Mathematical Magic Show, 1978. 146-159.

17. Harary F. The cell growth problem and its attempted solutions. Leipzig: Beitrage zur Grathen theory, 1968. 49-60.

18. Klarner D. A cell growth problems. Cand. J. Math. 19, 1967. 851-863.

19. Klarner D.A., Rivest R.L. A procedure for improving the upper bound for the number of n-ominoes. Canad. J. Math., 25, 1973. 585-602.

20. Knop J.V., Szymanski Z., Trinajstie N. On the total number of polygexes. Match: Commun. Math. Chem., 16, 1984. 119-134.

21. Maleev A.V. Algorithm and computer-program search for variantsof polyhexes packing in plane. Crystallography Reports, 60, 2015. 986-992.

22. Myers J. Polyomino, polygex and polyamond tiling. http://www.srcf.ucam. org/jsm28/tiling/.

23. Read R.C. Contributions to the cell-growth problem. Canad. J. Math., 14, 1962. 1-20.

24. Rhoads G.C. Planar tilings by polyominoes, polyhexes and polyamonds. Journal of Computational and Applied Mathematics, 174, 2005. 329-353.

25. Schattschneider D. Will it tile? Try the Conway criterion! Math. Magazine, $53: 4,1980.224-233$.

\title{
АЛГОРИТМЫ ПЕРЕБОРА ВАРИАНТОВ РЕШЁТЧАТЫХ И ПЕРИОДИЧЕСКИХ УПАКОВОК ТРЕХМЕРНЫХ ПОЛИКУБОВ
}

Серавкин К.Г., Малеев А.В., Шутов А.В.

https://doi.org/10.31241/MIEN.2018.14.12

Владимирский государственный университет им. А.Г. и Н.Г. Столетовых, Владимир

\section{Аннотация}

Одной из важных задач кристаллографии и кристаллохимии является выявление особенностей молекулярных упаковок в кристаллическом состоянии. В связи с этим возникает, с одной стороны, проблема математического описания взаимного расположения молекул в кристалле по возможности меньшим числом параметров, с другой стороны, задача априорного предсказания возможных кристаллических структур данного химического соединения. Именно эти две задачи призван решать предложенный в [5] и обобщённый в [3] метод дискретного моделирования молекулярных упаковок в кристаллах (МДМ). Метод основан на замене молекул их дискретными моделями - поликубами - и расчёте всех возможных вариантов расположения этих поликубов в дискретном упаковочном пространстве.

\section{Summary}

One of the main problems of crystallography and crystal chemistry is to reveal the peculiarities of molecular packings in the crystal state. In this regard, there is, on the one hand, the problem of the mathematical description of the relative placement of the molecules in the crystal with less as possible parameters, on the other hand, the problem 
of a priori prediction of possible crystal structures of particular chemical compound. Proposed in [5] and generalized in [3] the discrete modeling method of molecular packings in crystals is designed to solve quite this two problems. Discrete modeling method is based on the replacement of molecules by their discrete models - polycubes - and calculating all of the possible location of these polycubes in the discrete packaging space.

\section{Определения}

Поликуб - это геометрическая фигура со связной внутренней областью, состоящая из конечного числа элементарных ячеек простой кубической решётки $L=\square^{3}$.
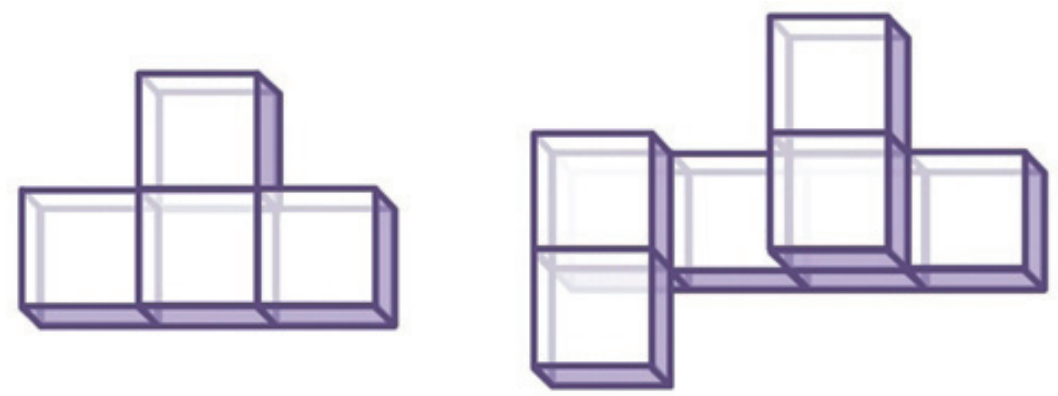

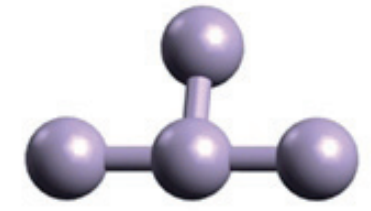

a) T-тетракуб

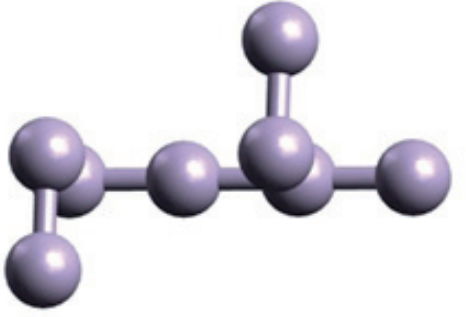

б) 2-анизоэдральный полукуб

Рис. 1. Представление поликубов в виде графа связности.

В классическом представлении поликубов слой, находящийся ближе к наблюдателю, практически полностью закрывает остальные слои. Для визуализации поликубов в пространстве удобнее использовать дуальное представление поликубов в виде графа связности: вершины графа есть центры ячеек поликуба, две вершины соединены ребром тогда и только тогда, когда две ячейки имеют общую грань. На рис. 1 приведён пример представления поликубов в виде графов связности: а) Т-тетракуб; б) наименьший 2-анизоэдральный поликуб, рассмотренный в работе Гамбини и Вуиллона [6].

Упаковка поликубов в пространстве - это такое расположение поликубов, при котором любые два поликуба не имеют общих внутренних точек. Упаковка поликубов называется нормальной, если любые две ячейки различных поликубов либо не имеют общих точек, либо имеют общую вершину, либо общее ребро, либо общую целую грань. В дальнейшем мы будем иметь дело только с нормальными упаковками. Тогда центры всех ячеек поликубов упаковки могут быть расположены в узлах решётки $L$. Упаковка поликубов называется периодической, если она обладает трансляционной симметрией, которая определяется некоторой трехмерной решёткой трансляций $\Lambda$, являющейся подрешёткой решётки $L$. 
Коэффициент упаковки поликубов может быть посчитан по формуле $k=p / N$, где $p$ - объем поликубов упаковки, попадающих в фундаментальную область решётки трансляций $\Lambda, N$ - объем этой фундаментальной области, выраженный в объемах ячеек поликубов. При $k=1$ упаковка поликубов является разбиением.

\section{Упаковочные пространства (УП)}

Любая невырожденная целочисленная $3 \times 3$ матрица $A$ задает в решётке $L$ подрешётку $\Lambda$. При этом будем считать, что базис подрешётки образуют векторы, составленные из столбцов матрицы $A$. То есть векторы $\mathbf{a}_{i}$ подрешётки $\Lambda$ можно выразить через векторы $\mathbf{v}_{i}$ решётки $L$ по формуле $\mathbf{a}_{i}=A \mathbf{v}_{i}$. Очевидно, что разные матрицы могут задавать одну и ту же подрешётку $\Lambda$. Для этого необходимо и достаточно, чтобы определители этих матриц были равными по абсолютной величине, а векторы-столбцы одной матрицы линейно выражались с целыми коэффициентами через векторы-столбцы другой. На языке матричной алгебры: две матрицы $A$ и $B$ задают одну и ту же подрешётку тогда и только тогда, когда $B=A U$, что равносильно равенству

$$
A^{-1} B=U,
$$

где $U$ - целочисленная унимодулярная матрица.

Соотношение (1) является отношением эквивалентности, разбивающим множество всех целочисленных матриц на непересекающиеся классы.

В работе [2] показано, что в каждом классе матриц, задающих одну и ту же подрешётку $\Lambda$ в трехмерной решётке $L$, существует единственная матрица вида:

$$
\left(\begin{array}{ccc}
a_{11} & a_{12} & a_{13} \\
0 & a_{22} & a_{23} \\
0 & 0 & a_{33}
\end{array}\right)
$$

где $a_{11}, a_{22}, a_{33}>0 ; 0 \leq a_{12}<a_{11} ; 0 \leq a_{13}<a_{11} ; 0 \leq a_{23}<a_{22}$,

Множество узлов подрешётки $\Lambda$, входящих в фундаментальный параллелепипед матрицы $A\left(\sum \theta_{i} a_{i i}, 0 \leq \theta_{i}<1, i=1 . .3,0 \leq \theta_{i}<1\right)$ называется упаковочным параллелепипедом. Число узлов, входящих в упаковочный параллелепипед, равно $N=|\operatorname{det} A|$ и называется порядком упаковочного пространства.

Припишем узлам решётки, входящим в упаковочный параллелепипед, различные веса. Например, веса от 0 до $N-1$ по формуле:

$$
g\left(x_{1}, x_{2}, x_{3}\right)=\sum_{i=1}^{3}\left(x_{i} \prod_{j=1}^{3} a_{j j}^{k(i, j)}\right), \text { где } k(i, j)=\left\{\begin{array}{l}
0, \text { если } i \leq j \\
1, \text { если } i>j
\end{array}\right.
$$

Периодически продолжив эти веса на всю решётку $L$, получим заданную на множестве точек решётки функцию $g\left(x_{1}, x_{2}, x_{3}\right)$, значения которой 
определяются выбранной в ней подрешёткой $\Lambda$. Решётка $L$, на которой задана функция весов $g\left(x_{1}, x_{2}, x_{3}\right)$ называется упаковочныл пространством (УП).
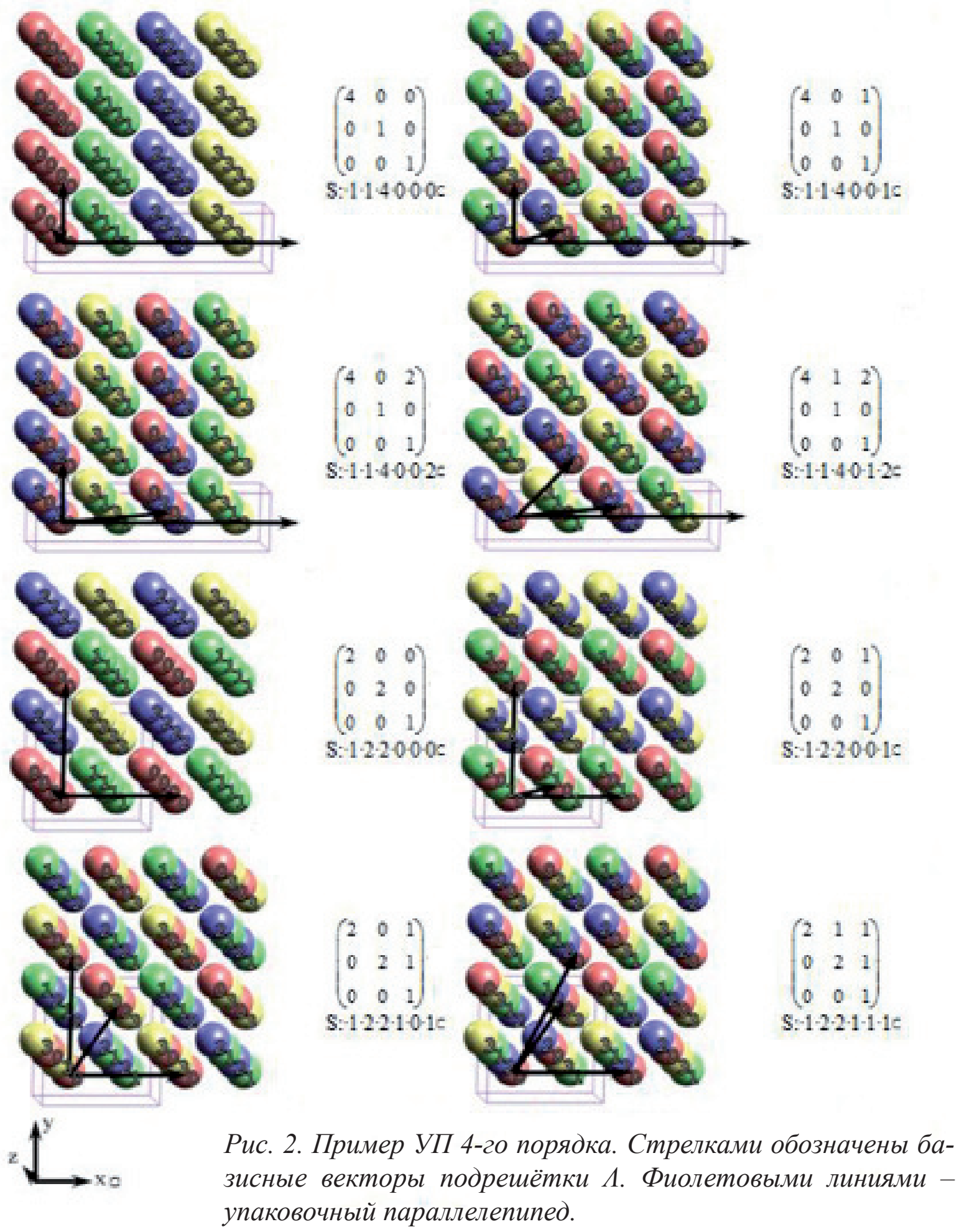

Матрица, задающая подрешётку $\Lambda$ решётки упаковочного пространства $L$ вида (2), называется матрицей упаковочного пространства. Узлы решётки, на которой задано УП, называются точками упаковочного пространства. Множество точек УП с одинаковыми весами образуют одну и ту же, с точностью до параллельного переноса, подрешётку $\Lambda$ исходной решётки $L$. 
Всего существует 35 УП 4 порядка. На рис. 2 приведены примеры некоторых из них.

Так как диагональные элементы матриц УП являются натуральными числами, а их произведение равно $N$, существует взаимно-однозначное соответствие между множествами диагональных элементов матриц УП и разложениями $N$ на 3 множителя. Очевидно, число различных таких разложений конечно.

Каждому (с учетом порядка следования сомножителей) разложению $N=\Delta_{1} \Delta_{2} \Delta_{3}$ соответствует ряд матриц УП, в которых диагональные элементы $a_{i i}=\Delta_{i}$, а элементы выше диагонали могут принимать любые целочисленные значения от 0 до $\Delta_{i}-1$. Общее число матриц УП порядка $N$ конечно и определяется суммированием по всем возможным разложениям числа $N$ на 3 множителя с учетом порядка множителей в разложении:

$$
\sum \Delta_{1}^{0} \Delta_{2}^{1} \Delta_{3}^{2}
$$

Число матриц УП порядка $N$ совпадает с числом $I_{3 . N}$ 3-мерных подрешёток 3-мерной решётки с индексом подрешётки $N$. Формула для расчёта $I_{3 . N}$ выведена в монографии [1].

\section{Независимые упаковочные пространства (НУП)}

Полная группа $O_{h}$ симметрий куба содержит $2^{3} 3$ ! элементов. Она определяет 48 матриц $P$ преобразований, переводящих простую кубическую решётку саму в себя. Два УП, заданные матрицами $A$ и $B$, называются связанHblмu, если хотя бы для одной из матриц $P$ преобразований решётки выполняется равенство:

$$
(P A)^{-1} B=U,
$$

где $U$ - целочисленная унимодулярная матрица. Отношение связанности является отношением эквивалентности и разбивает множество УП заданного порядка на непересекающиеся классы. Составим числовую последовательность из элементов матрицы УП: $S=\overline{a_{33} a_{22} a_{11} a_{23} a_{12} a_{13}}$. Последовательность $S$ позволяет упорядочить УП одного порядка. УП с наименьшим $S$ из всех УП одного класса называется независимым упаковочнылм пространством (НУП).

Для нахождения всех НУП данного порядка $N$ можно использовать следующий алгоритм:

1. Перебираем все матрицы УП $A$ заданного порядка $N$. Для каждой матрицы $A$ находим все связанные матрицы $A_{p}=P A$, приведенные к виду (2).

2. Сравниваем последовательности $S\left(A_{p}\right)$ и $S(A)$ : если для всех $A_{p}$ выполняется условие $S\left(A_{p}\right) \geq S(A)$, то УП $A$ является независимым по определению.

Матрицы всех НУП 4 порядка имеют следующий вид: 


$$
\begin{gathered}
\left(\begin{array}{lll}
4 & 0 & 0 \\
0 & 1 & 0 \\
0 & 0 & 1
\end{array}\right),\left(\begin{array}{lll}
4 & 0 & 1 \\
0 & 1 & 0 \\
0 & 0 & 1
\end{array}\right),\left(\begin{array}{lll}
4 & 0 & 2 \\
0 & 1 & 0 \\
0 & 0 & 1
\end{array}\right),\left(\begin{array}{lll}
4 & 1 & 1 \\
0 & 1 & 0 \\
0 & 0 & 1
\end{array}\right),\left(\begin{array}{lll}
4 & 1 & 2 \\
0 & 1 & 0 \\
0 & 0 & 1
\end{array}\right),\left(\begin{array}{lll}
4 & 2 & 2 \\
0 & 1 & 0 \\
0 & 0 & 1
\end{array}\right), \\
\left(\begin{array}{lll}
2 & 0 & 0 \\
0 & 2 & 0 \\
0 & 0 & 1
\end{array}\right),\left(\begin{array}{lll}
2 & 0 & 1 \\
0 & 2 & 0 \\
0 & 0 & 1
\end{array}\right)\left(\begin{array}{lll}
2 & 0 & 1 \\
0 & 2 & 1 \\
0 & 0 & 1
\end{array}\right) .
\end{gathered}
$$

Перебор различных вариантов упаковок поликубов достаточно проводить только для НУП. В остальных, связанных УП, реализуются те же упаковки с точностью до преобразований $P$.

На рис. 3 представлена зависимость отношения количества всех УП к числу НУП от порядка упаковочных пространств.

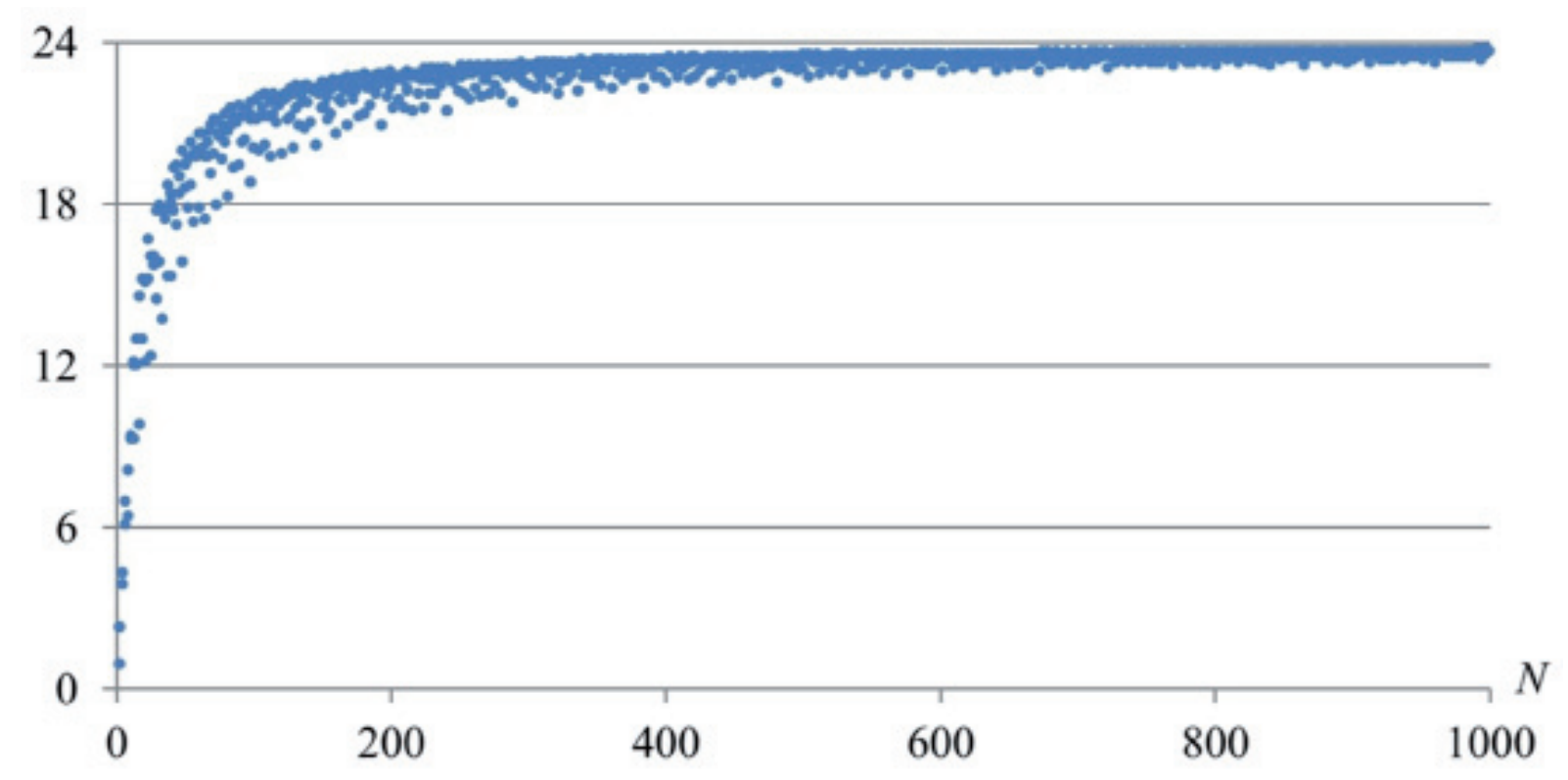

Рис. 3. Отношение количества всех УП к числу НУП.

\section{Точечная симметрия упаковочных пространств}

Точечным симметрийным преобразованием УП называется преобразование $P$, задающее взаимно-однозначное отображение упаковочного пространства на себя. Это означает, во-первых, что это преобразование переводит решётку, на которой задано УП, саму в себя; во-вторых, если какую-либо точку УП с весом $g_{1}$ это преобразование переводит в точку с весом $g_{2}$, то любую другую точку с весом $g_{1}$ это преобразование также переводит в точку с весом $g_{2}$. Для того, чтобы УП, заданное матрицей $A$, обладало точечным симметрийным преобразованием, заданным матрицей $P$, необходимо и достаточно чтобы выполнялось равенство:

$$
(P A)^{-1} A=U,
$$


где $U$ - целочисленная унимодулярная матрица.

Множество всех точечных симметрийных преобразований $P$ конкретного УП образуют группу, являющуюся подгруппой группы $O_{h}$.

Преобразование инверсии $I$, соответствующее смене знаков всех базисных векторов решётки

$$
I=\left(\begin{array}{ccc}
-1 & 0 & 0 \\
0 & -1 & 0 \\
0 & 0 & -1
\end{array}\right)
$$

является точечным симметрийным преобразованием любого УП. Действительно,

$$
(I A)^{-1} A=(-A)^{-1} A=-A^{-1} A=-E .
$$

\section{Кодировка периодической упаковки}

Каждый из кубов, составляющих поликубы, имеет 6 граней. Так как каждая грань принадлежит двум соседним кубам, то для того, чтобы задать границы упаковки в фундаментальном параллелепипеде достаточно для каждого куба фундаментального параллелепипеда указать входит ли в границы 3 из 6 его граней.

Отсутствие или наличие границы в направлениях, противоположных направлениям координатных осей, зададим цифрой $a_{i}$, равной соответственно 0 или 1. Последовательность $a_{i}$ определяет двоичную цифру кода упаковки $c(g)=\sum \alpha_{i} 2^{i-1}$, где $g-$ вес точки УП. Разряды цифры кода $a_{i}$ в двоичной системе содержат признак наличия или отсутствия грани в упаковке (см. рис. 4).
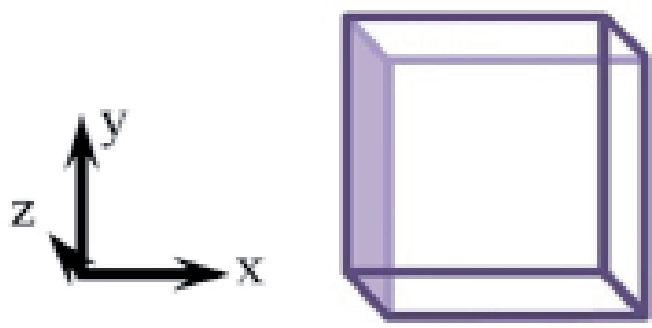

$$
c_{1}=\alpha_{1} 2^{0}
$$

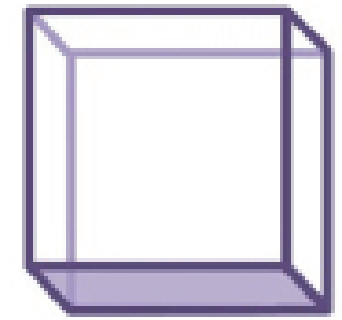

$$
c_{2}=\alpha_{2} 2^{1}
$$

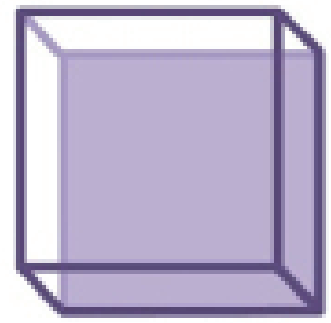

$$
c_{3}=\alpha_{3} 2^{2}
$$

Рис. 4. Формирование иифры кода упаковки в точке УП.

В упаковочном параллелепипеде содержатся $N$ точек УП, поэтому грани упаковки в фундаментальном параллелепипеде определяют последовательность цифр $\overline{c(0) c(1) \ldots c(N-1)}$ в восьмеричной системе исчисления, называемую кодом упаковки.

На рис. 5 представлена решётчатая упаковка Т-тетракуба в УП S: 114022 с кодом упаковки 6657. 
По коду упаковки и УП можно однозначно восстановить грани упаковки. По цифрам кода упаковки определяются грани в точках УП и транслируются в точки с соответствующими весами.

При таком определении, код упаковки зависит от ориентации базиса и положения начала координат. Поэтому, одной и той же, с точностью до движений, упаковке поликубов соответствует совокупность различных кодов упаковки. С целью решения проблемы однозначности кода упаковки, из всей этой совокупности кодов выбирается максимальный, который называется приведённылм. Например, приведённым кодом для кода 6657 в УП S: 114022 является код 7665.

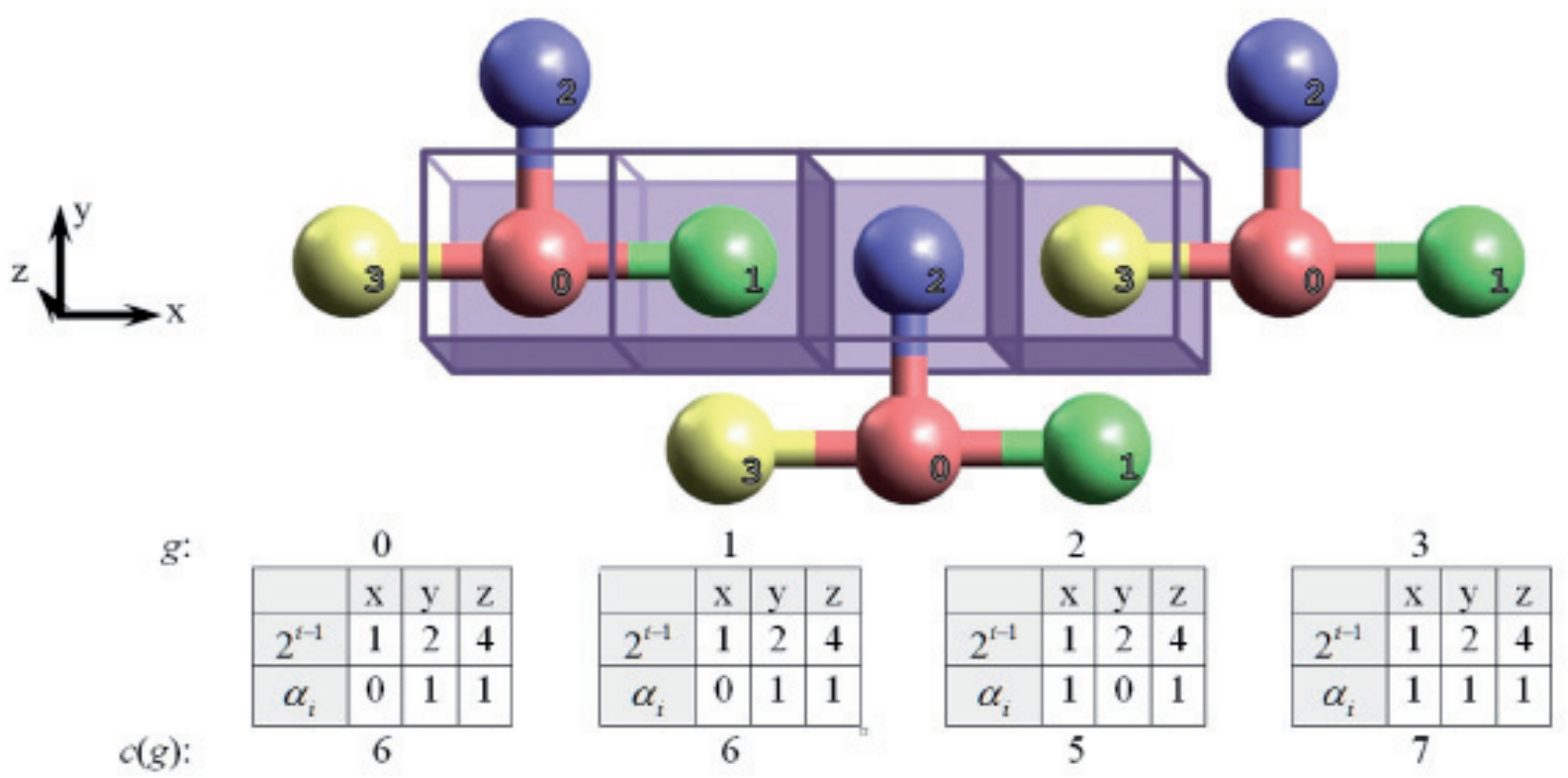

Рис. 5. Формирование кода упаковки по точкам УП. Код упаковки: 6657.

\section{Критерий существования решётчатой упаковки с одним поликубом на фундаментальную область решётки трансляций}

Назовем решётчатой упаковкой поликуба периодическую упаковку с одним поликубом в фундаментальном параллелепипеде подрешётки $\Lambda$. Задание поликуба сводится к заданию множества целочисленных векторстолбцов $\left\{\boldsymbol{\beta}_{i} \mid i=1,2, \ldots, r\right\}$ с координатами центров ячеек поликуба в базисе решётки $L$, где $r$ - количество ячеек поликуба. Тогда критерий существования решётчатой упаковки поликуба можно сформулировать в следующем виде [4]:

Для того, чтобы сущчествовала решётчатая упаковка заданного поликуба $\left\{\boldsymbol{\beta}_{i} \mid i=1,2, \ldots, r\right\}$ с коэффициентом упаковки $k=r / N$, необходимо и достаточно, чтобы существовало хотя бы одно УП порядка N, r точек которого с координатами $\left\{\boldsymbol{\beta}_{i}+\boldsymbol{x}_{0} \mid i=1,2, \ldots, r\right\}$ имеют попарно различные веса, где $\boldsymbol{x}_{0}$ - прочзвольный изелочисленный вектор.

На рис. 6 представлен результат проверки критерия решётчатой упаковки Т-тетракуба в некоторых УП. 


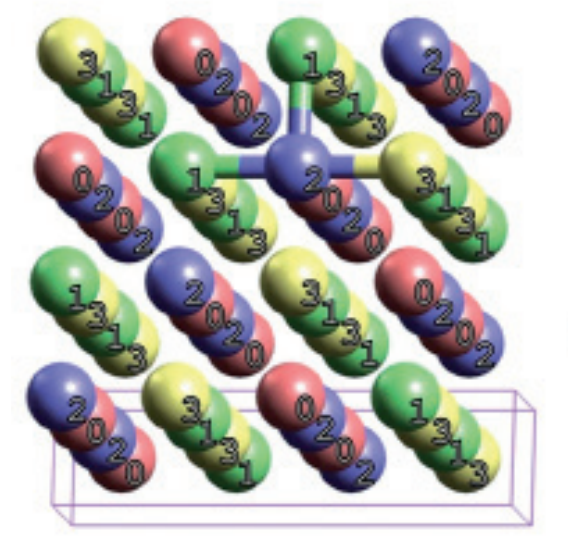

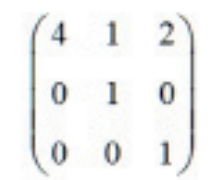

S: 114012

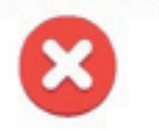

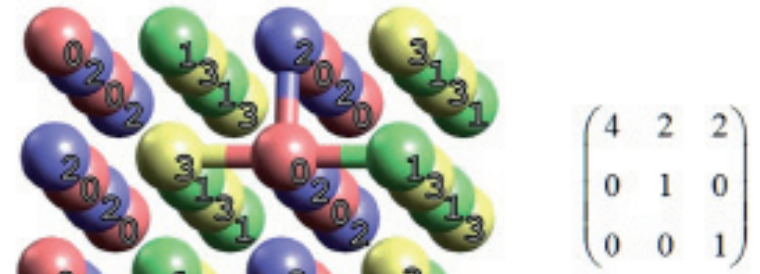

S: 114022

Рис. б. Проверка критерия решётчатой упаковки Т-тетракуба.

\section{Алгоритм перебора вариантов решётчатой упаковки с одним поликубом на фундаментальную область решётки трансляций}

Алгоритм перебора вариантов упаковки с одним заданным поликубом на фундаментальную область решётки трансляций включает в себя следующие этапы:

1. В качестве исходных данных задаём координаты ячеек поликуба, где $\mathrm{P}=\left\{\boldsymbol{\beta}_{i}+\boldsymbol{x}_{0} \mid i=1,2, \ldots, r\right\}$ и коэффициент упаковки $k=r / N$.

2. Добиваемся параллельным переносом, чтобы одна из точек поликуба имела координаты $(0,0,0)$. Такая точка называется особой точкой поликуба.

3. Определяем все УП порядка $N$.

4. Находим группу точечных симметрий каждого УП.

5. Перебираем варианты упаковок для каждого УП:

5.1. Проверяем для каждой УП критерий решётчатой упаковки.

5.2. Рассчитываем приведенный код упаковки для каждой полученной решётчатой упаковки с учетом симметрии УП и сравниваем на совпадение с кодами решений, полученных ранее для этого УП.

На рис. 7 представлены все решётчатые упаковки Т-тетракуба с приведенными кодами.

Критерий существования периодической упаковки с несколькими заданными поликубами на фундаментальную область решётки трансляций

Пусть система поликубов $(C П)$ из $M$ поликубов задаётся набором целочисленных координат вектор-столбцов центров ячеек поликубов $\left\{\boldsymbol{\beta}_{i j} \mid i=1,2, \ldots, r_{j} ; j=1,2, \ldots, M\right\}$, где $r_{j}$ - количество кубов в $j$-м поликубе системы, а общее число кубов в СП $R=\sum_{j=1}^{M} r_{j}$. Тогда критерий существования периодической упаковки СП с заданным объёмом фундаментальной области $N$ можно сформулировать следующим образом [4]:

Для того, чтобы сущзествовала периодическая упаковка СП, состоящчей из М поликубов, с объёмом фундаментальной области $N$, необходи- 
мо и достаточно, чтобы существовал набор векторов $\left\{\boldsymbol{x}_{0} \mid j=1,2, \ldots,{ }_{j}\right\}$ и хотя бы одно УП порядка $N$ такие, что, во-первых, множество $\left\{\boldsymbol{\beta}_{i j}+\boldsymbol{x}_{0 \mathrm{j}} \mid j=1,2, \ldots ., r_{j} ; j=1,2, \ldots ., M\right\}$ состояло из $R$ точек; во-вторых, точки УП с такими координатами имели попарно различные веса.

Легко видеть, что в этом случае коэффициентом упаковки $k=R / N$.

\section{Алгоритм перебора вариантов периодической упаковки с несколькими заданными поликубами на фундаментальную область решётки трансляций}

Алгоритм перебора вариантов упаковки с несколькими заданными поликубами на фундаментальную область решётки трансляций включает в себя следующие этапы:

1. В качестве исходных данных задаём набор из $M$ поликубов $\left\{\mathrm{P}_{\mathrm{j}} \mid \mathrm{j}=1,2, \ldots ., M\right\}$, где $\mathrm{Pj}=\left\{\beta_{i j} \mid i=1,2, \ldots, r_{j}\right\}$ и объём фундаментальной области $\mathrm{N}$.

2. Добиваемся параллельным переносом, чтобы одна из точек каждого поликуба имела координаты $(0,0,0)$.

3. Находим для каждого трансляционно-независимого поликуба все возможные ориентации.

4. Определяем все НУП порядка $N$.

5. Находим группу точечных симметрий каждого НУП.

6. Перебираем варианты упаковок для каждого НУП:

6.1. Комбинируя ориентации трансляционно-независимых поликубов, составляем все возможные СП для заданного набора поликубов.

6.1.1. Проверяем для каждой ориентации каждого трансляционнонезависимого поликуба критерий решётчатой упаковки. Ориентации поликуба, для которых критерий решётчатой упаковки выполняется, называются разрешёнными. Если хотя бы для одного поликуба количество разрешённых ориентаций равно нулю, то в данном НУП искомых упаковок не существует.

6.1.2. Перебираем все возможные наборы весов особых точек всех поликубов кроме первого $\left(g_{2}, g_{3}, \ldots, g_{\mathrm{M}}\right)$, вес особой точки первого поликуба принимаем равным нулю. По набору весов $\left(g_{1}, g_{2}, g_{3}, \ldots, g_{м}\right)$ рассчитываем набор $V=\left(x_{1}, x_{2}, x_{3}, \ldots, x_{s}\right)$ векторов фундаментальной области УП с весами $\left(g_{1}, g_{2}, g_{3}, \ldots, g_{\mathrm{M}}\right)$.

6.1.3. Для каждого из возможных наборов $V$ рассчитываем наборы весов точек поликубов $w_{i j}=g\left(\boldsymbol{\beta}_{i j}+\boldsymbol{x}_{0 j}\right)$, содержащие ровно $R$ весов для каждой разрешенной ориентации. Если в наборе $w_{i j}$ все веса попарно различны, соответствующие наборы векторов $V$ и ориентации поликубов задают искомую упаковку. 
7. Рассчитываем приведенный код упаковки для каждой полученной упаковки с учетом симметрии УП и сравниваем на совпадение с кодами решений, полученных ранее для этого УП.
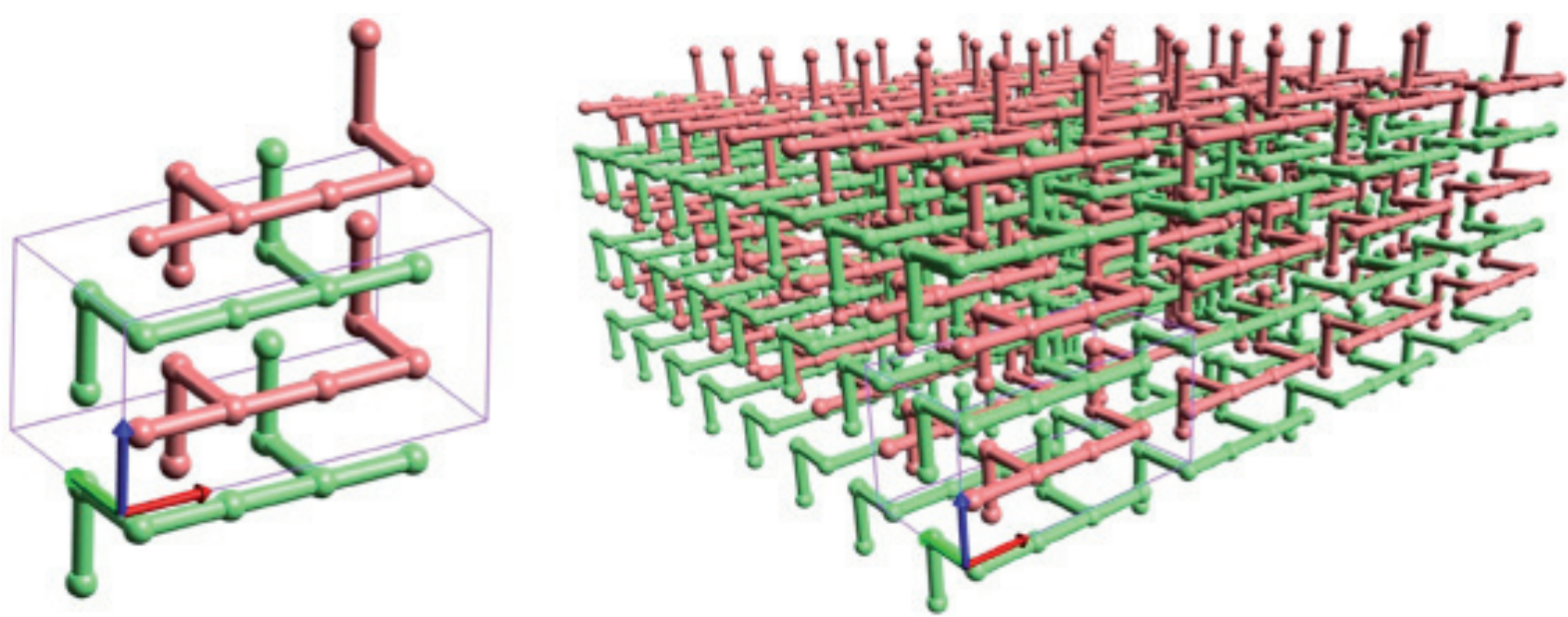

Рис. 8. Пример упаковки СП из двух 2-анизоэдральных поликубов. УП S: 224000. Код упаковки 7666713576661753.

На рис. 8 приведен пример упаковки набора из двух трансляционнонезависимых поликубов.

Работа выполнена при финансовой поддержке РФФИ, гранты 17-02-00835-а и 17-42-330787.

\section{Список литературы}

1. Делоне Б.Н., Фаддеев Д.К. Теория иррациональностей третьей степени // Тр. Матем. ин-та им. В. А. Стеклова. 1940. Т. 11. С. 3-340.

2. Касселс Дж. В. С. Введение в геометрию чисел. М.: Мир, 1965. 422 с.

3. Малеев А.В. $n$-Мерные упаковочные пространства // Кристаллография. 1995. T. 40. C. 394-396.

4. Малеев А.В. Структура кристаллов и квазикристаллов. Математическое моделирование кристал. и квазикристал. структур. Saarbrücken: Palmarium Academic Publishing, 2012. 328 c.

5. Малеев А.В., Рау В.Г., Потехин К.А., Пархомов Л.Г., Рау Т.Ф., Степанов С.В., Стручков Ю.Т. Метод дискретного моделирования упаковок в молекулярных кристаллах // Докл. АН СССР. 1990. Т. 315. № 6. С. 1382-1385.

6. Gambini I, and Vuillon L. Non lattice periodic tilings of R3 by single polycubes // Theoretical Computer Science. 2012. № 432. C. 52-57. 